\title{
Reviewing Hydrocephalus: Personal experience of a Neurosurgeon with Ventriculoperitoneal shunts treatment
}

\author{
Vinod K.S.Gautam ${ }^{1} *$, Ravinder Singh ${ }^{2}$, Sarbjeet Khurana ${ }^{3}$ \\ ${ }^{1}$ Assistant Professor, Department of Neurosurgery, Institute of Human Behaviour and Allied Sciences, Faculty of Medical Sciences, University \\ of Delhi, GNCT Delhi, Dilshad Garden, Delhi-110095 \\ ${ }^{2}$ Associate Professor \& Head, Department of Medical Anthropology, Institute of Human Behaviour and Allied Sciences, Faculty of Medical \\ Sciences, University of Delhi, GNCT Delhi, Dilshad Garden, Delhi-110095 \\ ${ }^{3}$ Associate Professor \& Head, Department of Epidemiology, Institute of Human Behaviour and Allied Sciences, Faculty of Medical Sciences, \\ University of Delhi, GNCT Delhi, Dilshad Garden, Delhi-110095 \\ *Corresponding author E-mail: drvksg@gmail.com
}

\begin{abstract}
Background: Hydrocephalus is one of the most common neurologic sequelae following insult to the central nervous system. The treatment of hydrocephalus is very challenging. Ventriculoperitoneal shunt surgery is the commonest form of CSF diversion procedure for hydrocephalus. The objective of this study is to look critically at the efficacy of this procedure and compare the outcome with other studies involving different therapeutic options for treating hydrocephalus.

Material \& Methods: Fourty three patients of hydrocephalus who were operated for ventriculoperitoneal shunt placement were included in the study. Review of medical records including operative notes, imaging studies and clinic-radiological follow up of all the patients were reviewed and analyzed.

Results: The common etiologies include post meningitic hydrocephalus, congenital hydrocephalus, NPH, posterior fossa tuberculomas, neurocysticercosis and posterior fossa tumor. Right sided ventriculoperitoneal shunt with medium pressure valve system was the commonest modality of treatment. None of the patients had any incidence of shunt infection.

Conclusion: The results of this retrospective study show that ventriculoperitoneal shunting is effective in the management of adult patients with obstructive hydrocephalus. The overall incidence of shunt revision was comparable with previously reported studies in the literature.
\end{abstract}

Keywords: Hydrocephalus, Ventriculoperitoneal Shunt.

\section{Introduction}

The introduction of various shunting and bypassing operations has transformed the management of hydrocephalus (Robertson, et al 1973). Most shunts drain the excess cerebrospinal fluid into the peritoneal cavity (ventriculo-peritoneal shunt), but alternative sites include the right atrium (ventriculo-atrial shunt), and pleural cavity (ventriculopleural shunt) (Low, et al 2010). Ventriculoperitoneal shunt surgery is commonest form of the CSF diversion procedures for hydrocephalus (Jha, et al 2010).

The development of shunt devices has greatly improved the survival and quality of life of patients with hydrocephalus (Low, et al 2010). In 1922, Dandy described an open ventriculostomy procedure performed via a lateral subtemporal approach, but the mortality rate was high (Dandy, 1945). In 1923, Mixter described the endoscopic perforation of the floor of the third ventricle, endoscopic third ventriculostomy (ETV), but it was not a success because of technical problems and complications (Mixter, 1923) and for many decades ETV was not used. Around 1960, the use of shunts became more common, as materials and techniques had improved. The use of modern shunts resulted in a reduction in mortality from $50 \%$ to about $10 \%$ (Hadenius, et al 1962).

The use of cranial endoscope for performing third ventriculostomy is now universally accepted especially for treating obstructive hydrocephalus. The success rate is variable considering different anatomical, etiological, and technical factors and selecting cases of hydrocephalus(Ali, et al 2013). External ventricular drainage (EVD) an effective therapy for treating rapidly progressive posthemorrhagic hydrocephalus and increased intracranial pressure. EVD is also a procedure of choice for CSF diversion in cases with ventriculitis. EVD can also be used as a temporary procedure for reducing ICP during neurosurgical procedure (Weninger, et al 1992).

Placement of ventriculoperitoneal shunt is a well-accepted neurosurgical procedure for the management of obstructive hydrocephalus (Reddy, et al 2012). Outcome following VP shunt surgery depends on many factors. Aim of this study is to determine the success rate of VP shunt for treating hydrocephalus and to analyze the factors which help in minimizing the complications related to this procedure.

\section{Material and methods}

This study was conducted at neurosurgery department of IHBAS, tertiary care neuropsychiatry hospital in Delhi, India. Forty three patients of hydrocephalus who were operated by the first author for ventriculoperitoneal shunt placement were included in the 
study by non-probabilty convenience sampling from January 2008 to October 2013. Study design was descriptive case series. Medical charts, operative notes, imaging studies, and clinical follow-up evaluations were reviewed and analyzed. Demographic details, clinical presentation, radiological features, indications for surgery, surgical findings and shunt related complications were analyzed for outcome evaluation.

All the patients were investigated systematically through various diagnostic tests; blood profile was evaluated with complete blood count. Cerebrospinal fluid examination comprising of the entire battery of biochemistry (proteins, sugar), cytology, gram stain , AFB stain, India ink stain and other investigations depending upon provisional diagnosis of the patients.

Radiological investigations like X-Ray chest, CT scan/MRI or both were done for all the patients. Neurosurgical procedure was done as per the required intervention according to the neurosurgical diagnosis of the patient and the evidence based practice. After neurosurgical intervention the patients were assessed radiologically and clinically in the follow up.

The data of the patients was entered, compiled and analysed in MS Excel and the statistical output was represented as frequencies and percentages. The tables representing the frequencies and percentages of the diagnosis and surgical procedures were made to summarize the findings.

\section{Results}

A total of 43 patients were included in the study. Age of the patients ranged from 4.5 months to 80 years. There were 23 male and 20 female patients. Detailed clinical assessment, preanesthetic checkup, hematological investigations and chest X-ray (posteroanterior view) were done in all patients. Cranial imaging investigations included Ultrasound, CT scan with or without contrast and MRI of the brain with or without contrast and MR spectroscopy. The common etiologies include post meningitic hydrocephalus (48.8\%), congenital hydrocephalus (11.6\%), NPH (9.3\%), Posterior fossa tuberculomas (9.3\%), Neurocysticercus (6.9\%), \& Posterior fossa tumor $(4.4 \%)$. Other causes were idiopathic, epidermoid, arachnoid cyst and trauma (Table-1). Per operative Ventricular tap was done to assess the depth of the ventricle from the cortical surface and the CSF pressure. CSF was collected in all the cases for cytology and biochemistry and other investigations depending upon the provisional diagnosis of the case. Right sided ventriculoperitoneal shunt with medium pressure valve system was the commonest modality of treatment. All four patients with normal pressure hydrocephalus were treated with low pressure right VP Shunt. One patient of idiopathic hydrocephalus had shown radiologic features of asymmetric hydrocephalus with marked left lateral ventricular enlargement. In this case left sided VP shunt medium pressure was done (Table -2). Two patients died during follow up due to non-surgical reasons. None of the patients had any incidence of shunt infection. Shunt related complications included chronic subdural hematoma in 2 cases, per rectal extrusion of peritoneal end in one case. One patient required shunt revision due to malpositioning of the ventricular end.

Table 1: Diagnostic Profile of the Study Group

\begin{tabular}{lll}
\hline Diagnosis & No of Cases & \% of Total \\
\hline Post Meningitic hydrocephalus & 21 & 48.8 \\
Congenital Hydrocephalus & 5 & 11.6 \\
Normal Pressure Hydrocephalus & 4 & 9.3 \\
Tuberculomas & 4 & 9.3 \\
Neurocysticercosis & 3 & 6.9 \\
Posterior Fossa Tumor & 2 & 4.4 \\
Arachnoid Cyst & 1 & 2.3 \\
Frontal Epidermoid & 1 & 2.3 \\
Trauma & 1 & 2.3 \\
Idiopathic & 1 & 2.3 \\
\hline
\end{tabular}


The majority of cases in most published series are infants with spina bifida or other malformations. In the series reported $41 \%$ of the patients were aged under 1 year, $25.9 \%$ were aged 1 to 15 years, and $30.0 \%$ were aged over 15 years, and meningitis and tumor were each as common a cause as myelomeningocele (Robertson et al. 1973). In our series, post meningitic hydrocephalus was the commonest cause of hydrocephalus $(48 \%)$ followed by congenital hydrocephalus (11\%).

The treatment of hydrocephalus is a challenging one (Low, 2010). In untreated hydrocephalus, death may occur by tonsillar herniation secondary to raised ICP with compression of the brain stem and subsequent respiratory arrest. Poor development of cognitive function in infants and children, or loss of cognitive function in adults can complicate untreated hydrocephalus. Visual loss can complicate untreated hydrocephalus and may persist after treatment (Jha, et al. 2010).

Some cases of hydrocephalus can be treated with short term medical treatment with carbonic anhydrase inhibitors, loop diuretic agents, osmotic agents and fibrinolytic therapy. The most suitable drug seems to be acetazolamide, alone or in combination with furosemide. Fibrinolytic therapy administered directly into the ventricular system in cases of post hemorrhagic hydrocephalus (Poca and Sahuquillo, 2005). Ventricular tapping, repeated lumbar punctures, subcutaneous reservoirs are others measures to treat hydrocephalus.

Surgical treatment is the preferred therapeutic option (Hamilton, 2009; Jha, et al 2010,). The commonly used surgical procedures are ventriculoperitoneal shunt, endoscopic third ventriculostomy and external ventricular drainage. The common complications are malposition (Misplacement) of ventricular end, shunt obstruction (blockage), shunt infection, disconnection, shunt migration, over drainage, hemorrhage (extradural, subdural and intracerebral hematoma), abdominal pseudocyst, and shunt extrusion.

Etiology of hydrocephalus has significant impact on the incidence of shunt revision or failure (Reddy et al 2011). Patients with tumors experience a significantly lower shunt revision rate than patients without tumors (Reddy et al 20012).

Maurice Choux et al proposed a protocol for shunt procedures involving modifications in the immediate pre-, intra-, and postoperative management of children undergoing shunt implantation to minimize high incidence of shunt infection, foremost problem of shunt implantation after mechanical malfunction. With implementation of new protocol, the incidence of shunt infection decreased dramatically from $15 \%$ to $0.33 \%$ (Maurice et al 1992). A study shows that a changing of gloves before handling the shunt catheter may be a simple and cost-effective way to reduce the burden of postoperative shunt infections (Rehman, et al 2010).

The selection of shunt procedure for patient depends on clinical features and radiological investigations. The right sided shunt surgery is commonly done and in some case left side VP shunt is inserted depending upon the diagnosis. For example, if patient has right sided cerebellopontine angle tumor and right sided retromastoid craniectomy is planned for excisison of the tumor, then in this case left VP shunt is done for treating hydrocephalus. Similarly, in patient with obstructive hydrocephalus with left sided lateral ventricular enlargement, left VP shunt is done. Usually in patients with obstructive hydrocephalus due to meningitis ventricular tap is done to send CSF for cytology, biochemistry and other investigations depending upon provisional diagnosis. If CSF investigations reveal infection, the external ventricular drain is inserted. If CSF study does not show any feature of meningitis, then VP shunt is done. Type of pressure of the valve of the shunt is decided on the basis of CSF pressure on ventricular tap. Low pressure VP shunt should not be done in children with obstructive hydrocephalus due to aqueductal stenosis. It can lead to overdrainage and subdural hematoma. So, for these patients with congenital hydrocephalus, a medium pressure right VP shunt is most appropriate. In patients of NPH (Normal Pressure Hydrocephalus), low pressure VP shunt is done.

Complications of the shunt can be avoided by taking simple measures like
- Preoperative part preparation, draping,

- Prophylactic antibiotic,

- Minimizing movements of many persons inside the operation theater, c

- Selecting appropriate type of the shunt,

- Securing shunt assembly of the shunts,

- Collection of the CSF during the surgery,

- Minimal handling of the shunt assembly,

- Changing of the glove prior to the handling of shunt, antibiotic therapy after insertion of shunt, etc.

If due care and preventive efforts are taken at every stage of shunt surgery, the frequency of shunt revision can be minimized. In case of failure is suspected, patient should again be investigated thoroughly before reoperation. As long as the ventricular tip is inside the ventricle it is very unlikely that shunt is not functioning. Shunt should not be revised if the ventricular end of the shunt has crossed to the opposite side lateral ventricle piercing the septum pellucidum.

\section{Conclusion}

Ventriculoperitoneal shunting is the most widely used neurosurgical procedure for the management of hydrocephalus. Endoscopic third ventriculostomy is another alternative to treat obstructive hydrocephalus. The results of study show that ventriculoperitoneal shunting is effective in the management of adult patients with obstructive hydrocephalus. The overall incidence of shunt revision was comparable with previously reported studies in the literature. The high incidence of shunt failure can be minimized by meticulous clinical work up, CSF study, selection of appropriate type of shunt, use prophylactic antibiotic, and precautions at the time of surgery.

\section{References}

[1] Robertson J.S., Maraqa M.I., Jennett Bryan: Ventriculopperitoneal shunting for hydrocephalus. British Medical Journal, 1973, 2, 289-292. http://dx.doi.org/10.1136/bmj.2.5861.289.

[2] Low D, Drake JM, Seow WT, Ng WH. Management of ventriculoperitoneal shunts in the paediatric population. Asian J Neurosurg. 2010 Jan; 5(1):7-14.

[3] Jha R, Khadka N, Kumar P.Management of Hydrocephalus - Our Experience. PMJN, Vol 10, Number 2, Jul-Dec 2010, 68-70.

[4] Dandy W E. Diagnosis and treatment of strictures of the aqueduct of Sylvius (causing hydrocephalus). Arch Surg 1945; 51:1-14. http://dx.doi.org/10.1001/archsurg.1945.01230040004001.

[5] Mixter WJ. Ventriculoscopy and puncture of the floor of the third ventricle. Boston Med Surg J 1923; 188:277-278. http://dx.doi.org/10.1056/NEJM192303011880909.

[6] Hadenius AM, Hagberg B, Hyttnäs-Bensch K, Sjögren I. Congenital hydrocephalus. II. Long-term prognosis of untreated hydrocephalus in infants. Nord Med 1962; 68:1515-9.

[7] Ali M, Usman M, Khan Z, Khan K.M.,Hussain R,and Khanzada K: Endoscopic third ventriculostomy for obstructive hydrocephalus. Journal of the College of Physicians and Surgeons, Pakistan 2013 , Vol. 23(5): 338-341.

[8] Weninger M, Salzer HR, Pollak A, Rosenkranz M, Vorkapic P, Korn A, Lesigang C. External ventricular drainage for treatment of rapidly progressive posthemorrhagic hydrocephalus. Neurosurgery. $1992 \mathrm{Jul}$; 31(1):52-7; discussion 57-8. http://dx.doi.org/10.1227/00006123199207000-00008.

[9] Reddy G., Shi R, and Nanda A., Guthikonda B. Obstructive hydrocephalus in adult patients: the Lousiana State University Health Sciences Center- Shreveport experience with ventriculoperitoneal shunts. Clin Neurol Neurosurg. 2012 Nov; 114(9):1211-6. http://dx.doi.org/10.1016/j.clineuro.2012.02.050.

[10]Eva -Karin Persson (2007) Hydrocephalus In Children Epidemiology and outcome. Published Thesis. Göteborg University Sweden Göteborg ISBN 978-91-628-7050-8 https://gupea.ub.gu.se

[11]Poca MA, Sahuquillo J. Short-term medical management of hydrocephalus. Expert Opin Pharmacother. 2005 Aug; 6(9):1525-38. http://dx.doi.org/10.1517/14656566.6.9.1525. 
[12]Hamilton MG. Treatment of hydrocephalus in adults.Semin Pediatr $\begin{array}{lll}\text { Neurol. } & \text { Mar } & \text { 2009; }\end{array}$ http://dx.doi.org/10.1016/j.spen.2009.02.001.

[13]Reddy GK, Bollam P, Shi R, Guthikonda B, Nanda A. Management of adult hydrocephalus with ventriculoperitoneal shunts: long-term single-institution experience. Neurosurgery. 2011 Oct; 69(4):774-80 http://dx.doi.org/10.1227/NEU.0b013e31821ffa9e.

[14]Maurice Choux, Lorenzo Genitori, Dorothy Lang, and Gabriel Lena. Shunt implantation: reducing the incidence of shunt infection. Journal $\begin{array}{llll}\text { of Neurosurgery } & 1992 ; & 77: 6, & 875-880\end{array}$ http://dx.doi.org/10.3171/jns.1992.77.6.0875

[15]Rehman AU, Rehman TU, Bashir HH, Gupta V. A simple method to reduce infection of ventriculoperitoneal shunts. J Neurosurg Pediatr. 2010 Jun; 5(6):569-72 http://dx.doi.org/10.3171/2010.2.PEDS09151. 